\title{
Artificial Detection of Lower-Frequency Periodicity in Climatic Studies by Wavelet Analysis Demonstrated on Synthetic Time Series ${ }^{\mathscr{O}}$
}

\author{
ASSAF HOCHMAN \\ Karlsruhe Institute of Technology, Institute of Meteorology and Climate Research, \\ Eggenstein-Leopoldshafen, Germany \\ HADAS SAARONI \\ Department of Geography and the Human Environment, \\ Porter School of the Environment and Earth Sciences, Tel Aviv University, Tel Aviv, Israel \\ FELIX ABRAMOVICH \\ Department of Statistics and Operations Research, School of Mathematical Sciences, \\ Tel Aviv University, Tel Aviv, Israel \\ PINHAS ALPERT \\ Department of Geophysics, Porter School of the Environment and Earth Sciences, Tel Aviv University, Tel Aviv, Israel
}

(Manuscript received 7 February 2019, in final form 21 July 2019)

\begin{abstract}
The continuous wavelet transform (CWT) is a frequently used tool to study periodicity in climate and other time series. Periodicity plays a significant role in climate reconstruction and prediction. In numerous studies, the use of CWT revealed dominant periodicity (DP) in climatic time series. Several studies suggested that these "natural oscillations" would even reverse global warming. It is shown here that the results of wavelet analysis for detecting DPs can be misinterpreted in the presence of local singularities that are manifested in lower frequencies. This may lead to false DP detection. CWT analysis of synthetic and real-data climatic time series, with local singularities, indicates a low-frequency DP even if there is no true periodicity in the time series. Therefore, it is argued that this is an inherent general property of CWT. Hence, applying CWT to climatic time series should be reevaluated, and more careful analysis of the entire wavelet power spectrum is required, with a focus on high frequencies as well. A conelike shape in the wavelet power spectrum most likely indicates the presence of a local singularity in the time series rather than a DP, even if the local singularity has an observational or a physical basis. It is shown that analyzing the derivatives of the time series may be helpful in interpreting the wavelet power spectrum. Nevertheless, these tests are only a partial remedy that does not completely neutralize the effects caused by the presence of local singularities.
\end{abstract}

\section{Introduction}

Spectral methods such as the continuous wavelet transform (CWT; frequently named wavelet analysis) and the fast Fourier transform have a special appeal for

Supplemental information related to this paper is available at the Journals Online website: https://doi.org/10.1175/JAMC-D-180331.s1.

Corresponding author: Assaf Hochman, assafhochman@ yahoo.com climate and paleoclimate research because they can be used to detect periodicities in time series. Many other applications of CWT were presented in the literature, including, for example, in the research fields of medicine, engineering, and finance (Addison 2017). CWT has therefore become a common tool for the study of signals within climate time series, with the capability to analyze them at different time scales, which is imperative in climate reconstruction and prediction (Torrence and Compo 1998). Moreover, an appealing feature of CWT is its ability to analyze nonstationary time series, which is vital in climate research (Lau and Weng 1995). 
Recently, CWT was used in a plethora of climate and geophysical studies (e.g., Gray et al. 2004; Knight et al. 2005; Li et al. 2011; DeLong et al. 2012; McCabeGlynn et al. 2013; Pike et al. 2013; Cox et al. 2014; Duan et al. 2014; Kreppel et al. 2014; Magee et al. 2014; Soon et al. 2014; Xu et al. 2014; Burn and Palmer 2015; Lee et al. 2015; Wright et al. 2015; Novello et al. 2016; Sharma et al. 2016). These studies have incorporated CWT as a primary tool, chosen from a variety of other signal analysis tools within the frequency domain. All of the above studies highlighted dominant periodicities (DPs) in climatic, paleoclimatic, and geophysical time series, attributing them to natural origins, such as the NAO, ENSO, and Pacific decadal oscillation (PDO); solar cycles; solar irradiance; thermohaline circulation; and others. They stressed that low-frequency DPs may suggest a long-term climatic process as opposed to local high-frequency features. Thus, low-frequency DPs were appealing in improving predictability of the climate system. However, conelike shapes that express lowfrequency DPs in the wavelet power spectrum that emerged in the aforementioned studies have motivated the present work.

In most studies, DPs were typically detected by a pointwise significance testing procedure applied to the wavelet spectrum (Torrence and Compo 1998). DPs are regarded herein as a statistically significant region of the wavelet spectrum within a band of lower frequencies/periodicities (e.g., Fig. 1), where it was assumed that such higher-power regions extending in time are not related to local features. As such, many climatic studies assigned DPs to natural cycles of the climate system, as mentioned above. However, applying pointwise testing procedures to simultaneous testing of a large number of wavelet coefficients ignores the severe multiple testing problem. Thus, it does not control the overall significance at the required level (e.g., $5 \%$ ) and, therefore, typically leads to detection of spurious patches in the wavelet power spectrum (e.g., Abramovich and Benjamini 1995; Maraun and Kurths 2004; Maraun et al. 2007). Nevertheless, pointwise significance testing is still the most commonly used significance test in climate studies.

Several improved significance testing procedures were considered in the literature. Maraun et al. (2007) proposed an areawise significance test. However, the main disadvantage of this test is the complexity of the significance level calculation, which involves a rootfinding algorithm. Liu et al. (2007) have addressed the bias problem in the estimate of the wavelet spectra in atmospheric and oceanic datasets. They have suggested a rectification procedure, which is the transform coefficient squared divided by the scale with which it associates. Schulte et al. (2015) developed a geometric method for significance testing in the wavelet domain. They found that this method produces results similar to the areawise significance test while being more computationally flexible and efficient. In a most recent study, the geometric method was improved by a cumulative areawise significance testing procedure (Schulte 2016). It was shown that the latter test implies higher statistical power in most cases, especially when the signal-to-noise ratio is high.

The purpose of this paper is to show that the CWT, even after applying the aforementioned state-of-the-art methods, still often identifies artificial lower-frequency DPs, caused by local singularities in a time series, which can lead to misinterpretation of the wavelet power spectrum. This observation is particularly important because of the enormous increase in the number of publications that are using wavelet analysis in climate research, from 15 publications per year in 1998 to about 550 in 2018, as revealed by a search of ScienceDirect (https://www.sciencedirect.com/).

\section{Data and methods}

To demonstrate the detection of artificial DPs in the wavelet power spectrum we applied the state-of-the-art cumulative areawise (Schulte 2016) and the geometric (Schulte et al. 2015) significance testing procedures (https://www.mathworks.com/matlabcentral/ fileexchange/) to six synthetic and four real climate time series, as follows:

1) a sine time series with an about 200-time-step lowfrequency period (Fig. 1),

2) the sunspot number time series (SILSO World Data Center 2019; Fig. 2),

3) a local abrupt change (step) time series that was generated manually (Fig. 3),

4) the stratospheric aerosol optical depth time series (Bourassa et al. 2012; Fig. 4),

5) a red-noise time series (Fig. 5),

6) the local abrupt change time series added to the red-noise time series (Fig. 6),

7) the sine time series added to the red-noise time series (Fig. 7),

8) the PDO reconstruction (Mann et al. 2009; Fig. 8),

9) the daily Niño-3.4 index for 12 July 2015-6 April 2018 (Reynolds et al. 2007; Fig. 9), and

10) a time series that contains a few local abrupt changes added to the red-noise time series (Fig. 10).

For all of these examples, we applied the advanced significance testing procedures of Schulte et al. (2015) 


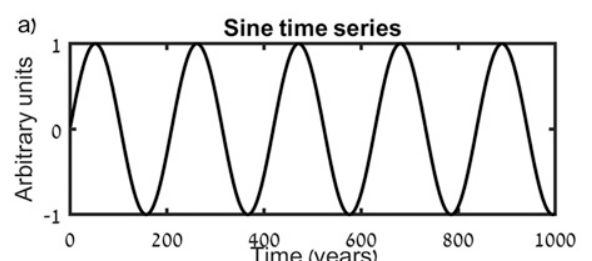

C) Cumulative Area Wise significance testing
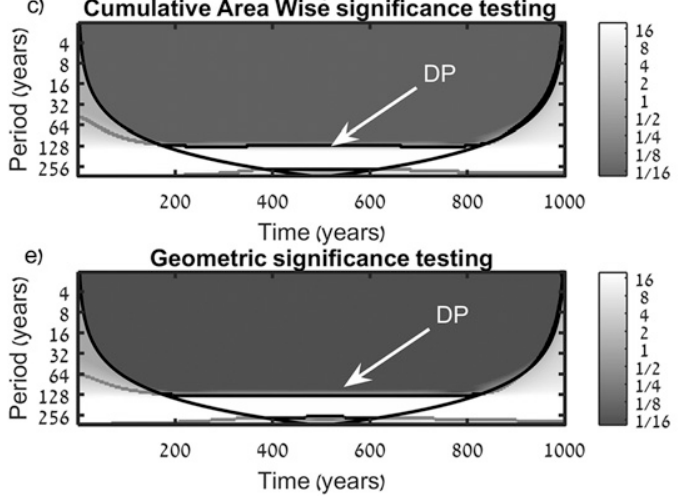
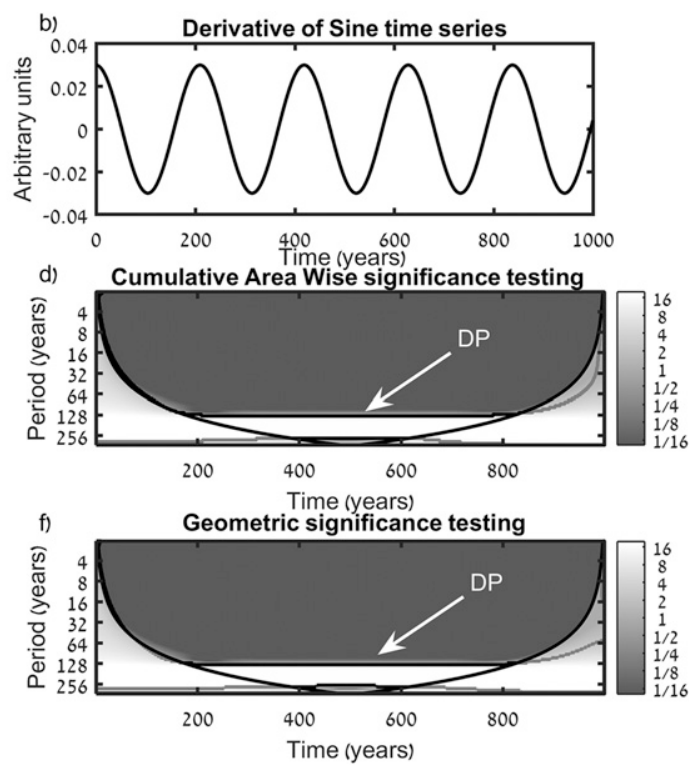

FIG. 1. (a) A sine time series and (b) the derivative of the sine time series (b) CWT applied with (c),(d) cumulative areawise (Schulte 2016) and (e),(f) geometric (Schulte et al. 2015) significance testing procedures using a Morlet 6 mother wavelet. The wavelet power spectrum is shown for both significance testing methods. The black contours are regions found to be significant at the $5 \%$ level with respect to a red-noise background using 1000 realizations from a Monte Carlo experiment. The shaded regions mark the cone of influence in which boundary conditions become important. The dominant periodicities (label DP) are marked by arrows. DPs are a statistically significant region of the wavelet spectrum within a band of lower frequencies/periodicities. It is emphasized that these higherpower regions extend in time and are not very local features in the power spectrum.

and Schulte (2016), in which we used the Morlet 6 "mother" wavelet, which is the most commonly used wavelet in geophysics. All time series were padded with zeros at the edges as typically recommended in geophysics. We modeled the background noise by a first order autoregressive process (red noise), which is typical in geophysics (Torrence and Compo 1998; Maraun et al. 2007; Schulte et al. 2015; Schulte 2016). The autocorrelation coefficient was estimated by the standard method of Allen and Smith (1996). To perform the significance
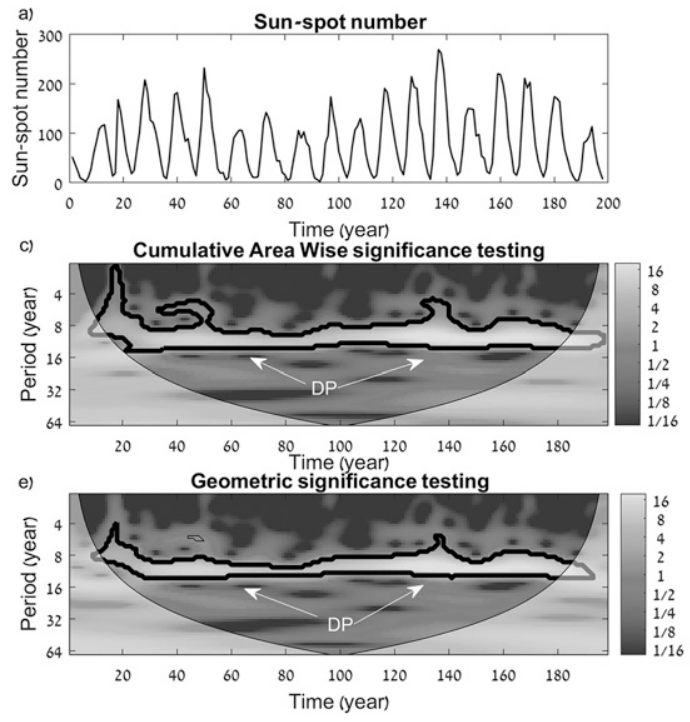
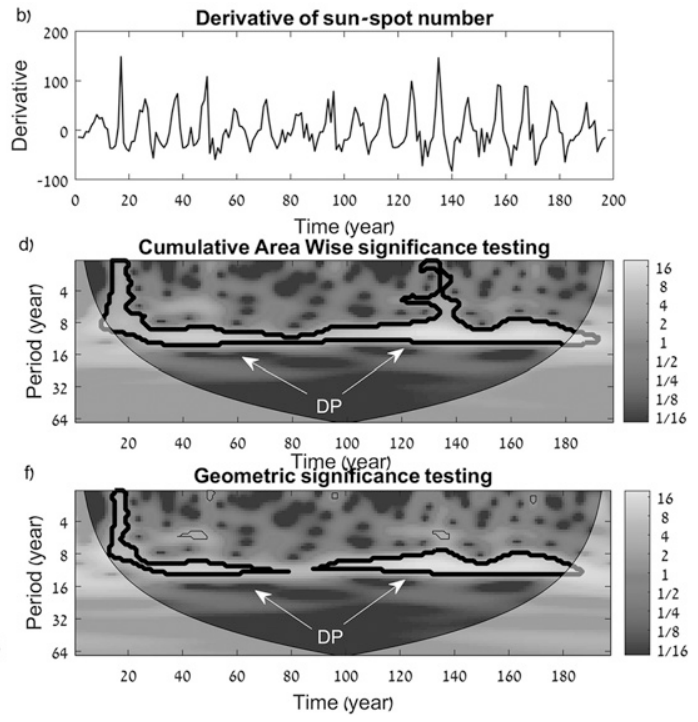

FIG. 2. As in Fig. 1, but for the sunspot number time series (SILSO World Data Center 2019). 

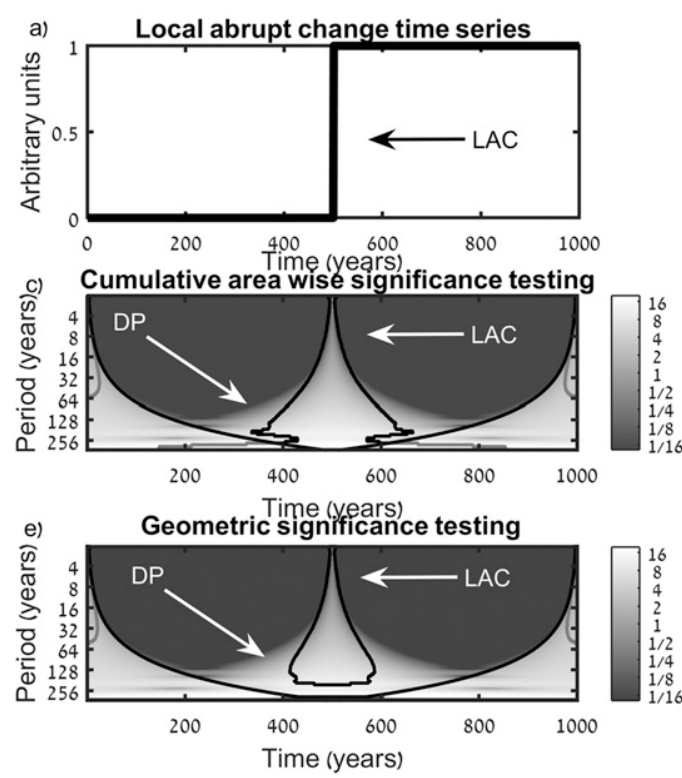
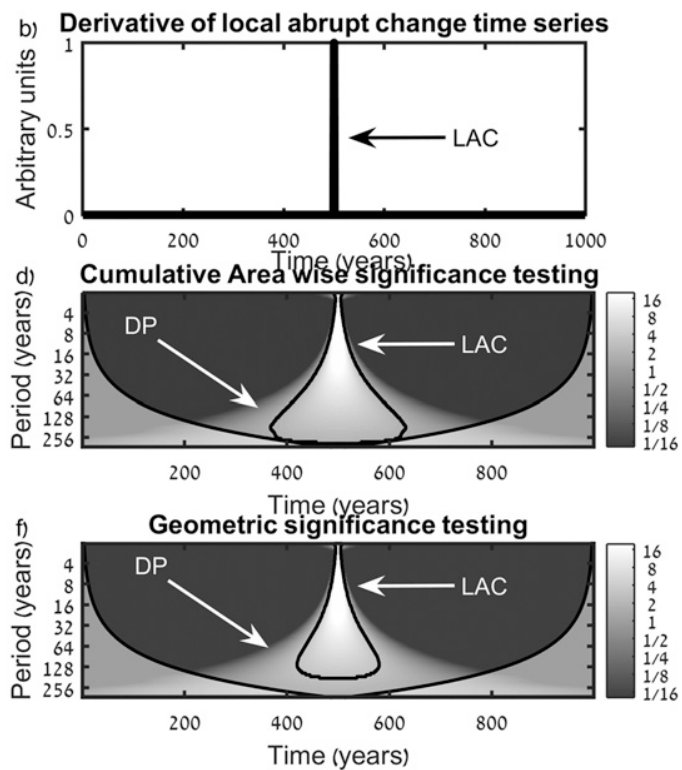

FIG. 3. As in Fig. 1, but for a local abrupt change time series. Local abrupt changes (label LAC) are marked by arrows.

testing procedures, we generated 1000 realizations of red-noise series, using Monte Carlo techniques, to estimate the threshold that guarantees the required $5 \%$ significance level. Note that the null distribution of the normalized area of a significance patch depends on the choice of null hypothesis (the time series is similar to a red noise background). For red-noise processes, the normalized area increases with increasing lag-1 autocorrelation coefficients (Schulte et al. 2015).
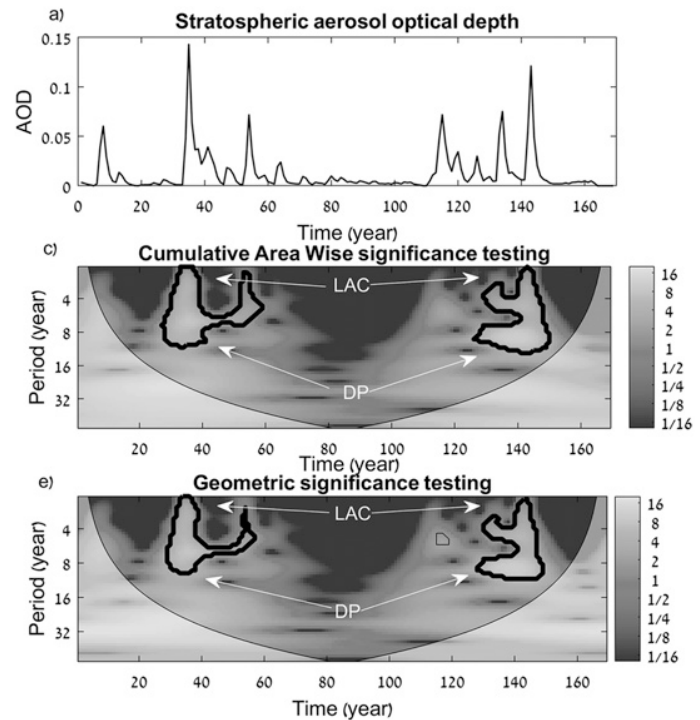

For the sake of comparison, the fast Fourier transform and pointwise wavelet significance testing (Torrence and Compo 1998) were also applied to the time series described above (Figs. S1 and S2, respectively, in the online supplemental material). A bias rectification procedure (Liu et al. 2007) was further applied to the synthetic time series before using the cumulative areawise significance test (Schulte 2016; online supplemental Fig. S3).
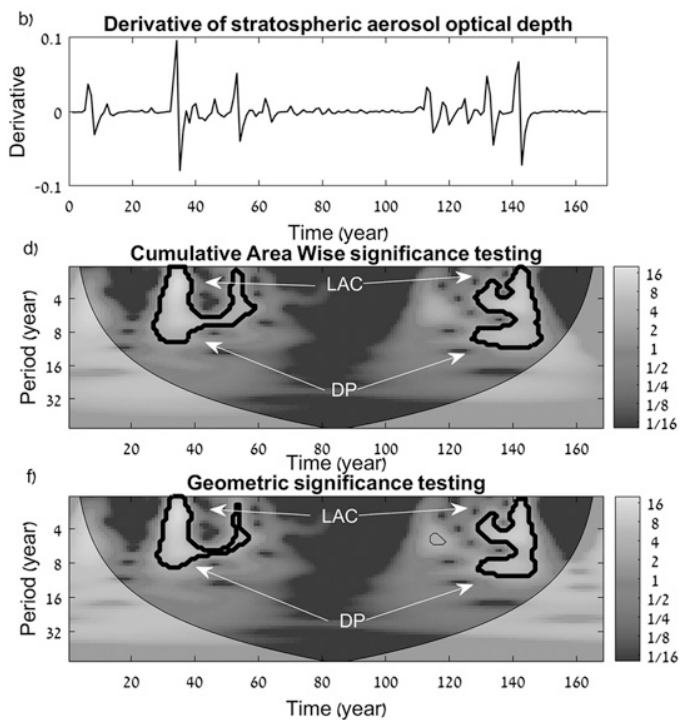

FIG. 4. As in Fig. 3, but for the stratospheric aerosol optical depth time series (Bourassa et al. 2012). 

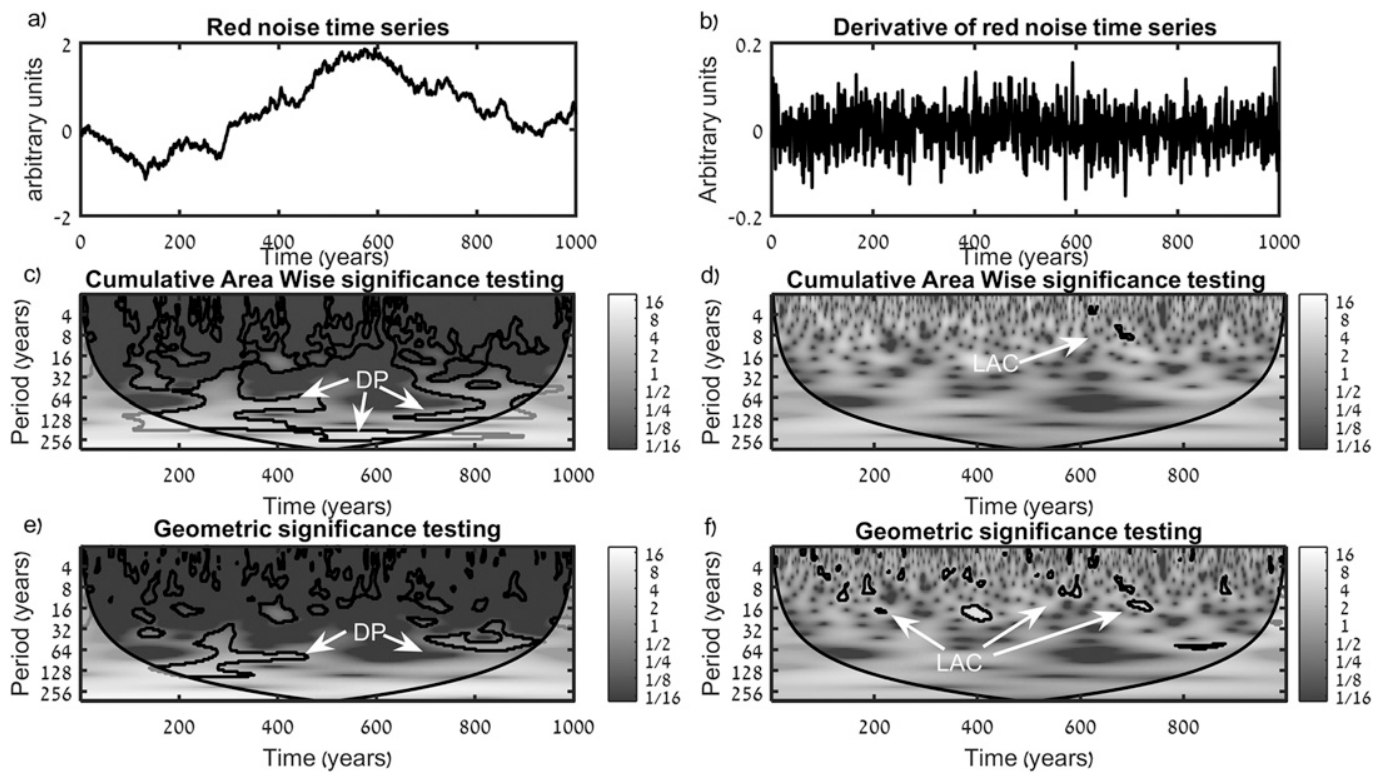

FIG. 5. As in Fig. 3, but for a red-noise time series.

Various boundary corrections may introduce an additional source of uncertainty in wavelet analysis of climate and other time series. Several options have been presented in the literature. For example, Lin and Franzke (2015) and Gallegati (2018) used symmetric/antisymmetric extensions of the original time series. However, no clear recommendation has yet been given for climate time series. Figure S4 presents the effect of using zero padding for the synthetic time series. In addition, the abovementioned time series were analyzed using other mother wavelets-for example, the Paul (Fig. S5 from the online supplemental material) and "Dog" ("derivative of Gaussian"; supplemental Fig. S6) mother wavelets.

To better distinguish between real DPs and DPs arising from local abrupt changes, wavelet-based tests were applied to the derivative (the rate of change at each time step) of the original time series. Taking the
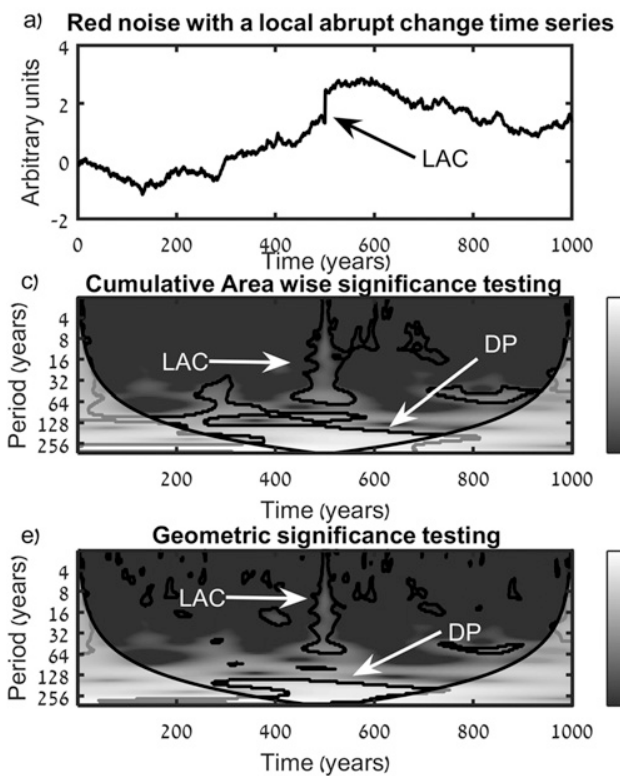
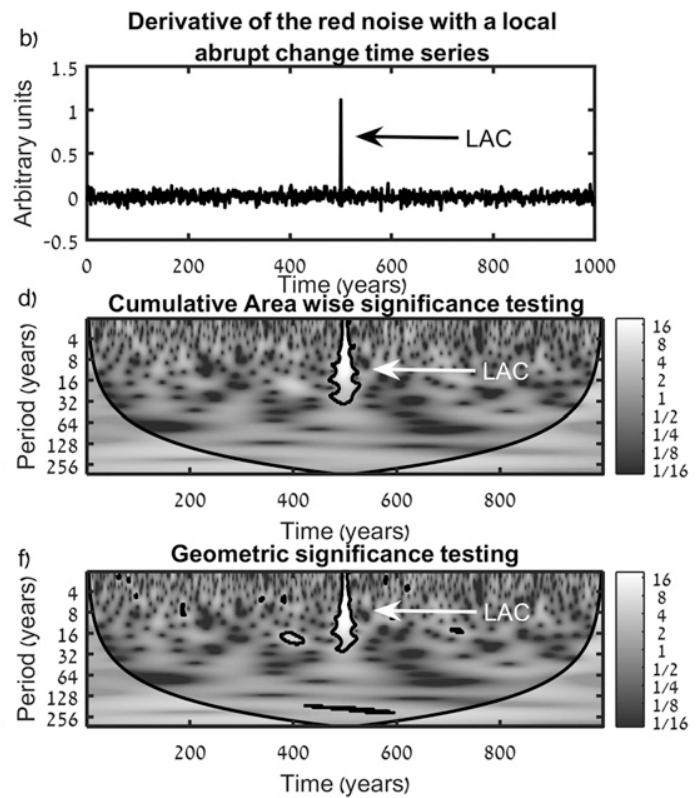

FIG. 6. As in Fig. 3, but for the red-noise time series added to the LAC time series. 

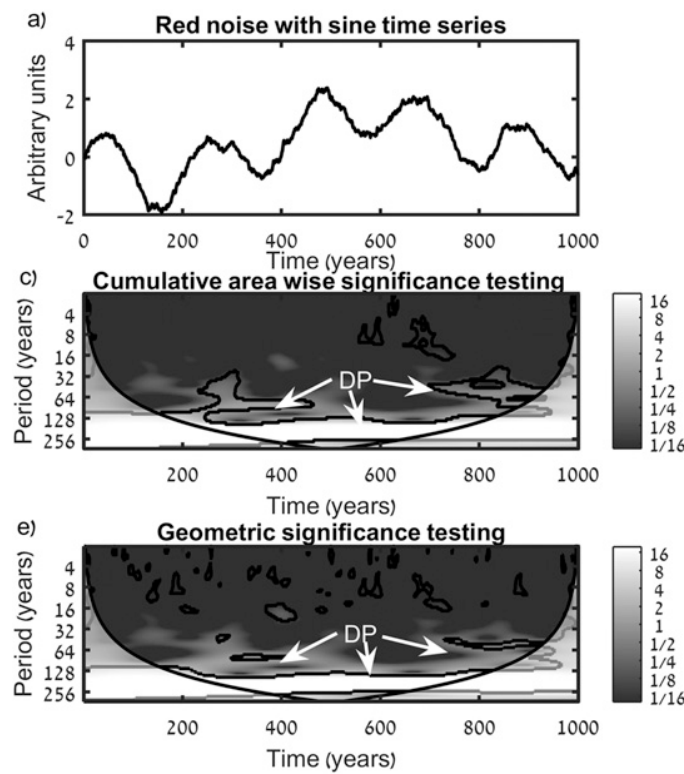
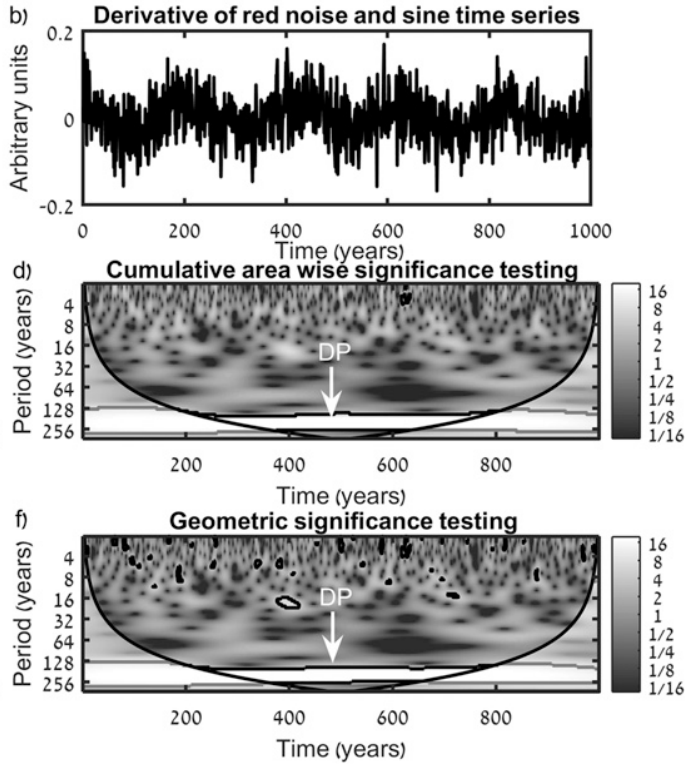

FIG. 7. As in Fig. 1, but for the sine time series added to the red-noise time series.

derivative does not affect periodicity. On the other hand, it reduces regularity of a signal, and a local singularity would be more strongly manifested at higher frequencies of the wavelet spectrum. See Mallat (2008, section 6) for rigorous mathematical analysis.

\section{Results}

CWT should only detect periodicities in the sine time series (Fig. 1), the sunspot number time series (Fig. 2;
SILSO World Data Center 2019), and the sine time series with red noise (Fig. 7) as shown in the fast Fourier transform analysis (supplemental Fig. S1). However, the wavelet power spectrum indicates the presence of DPs in all considered time series (Figs. 1-10). The detected periodicities in the wavelet power spectrum in Figs. 3-6 and $8-10$ are artificial and are caused by local abrupt changes in the time series. However, we stress that local abrupt changes may represent a real physical change in the system as, for example, in the time series of
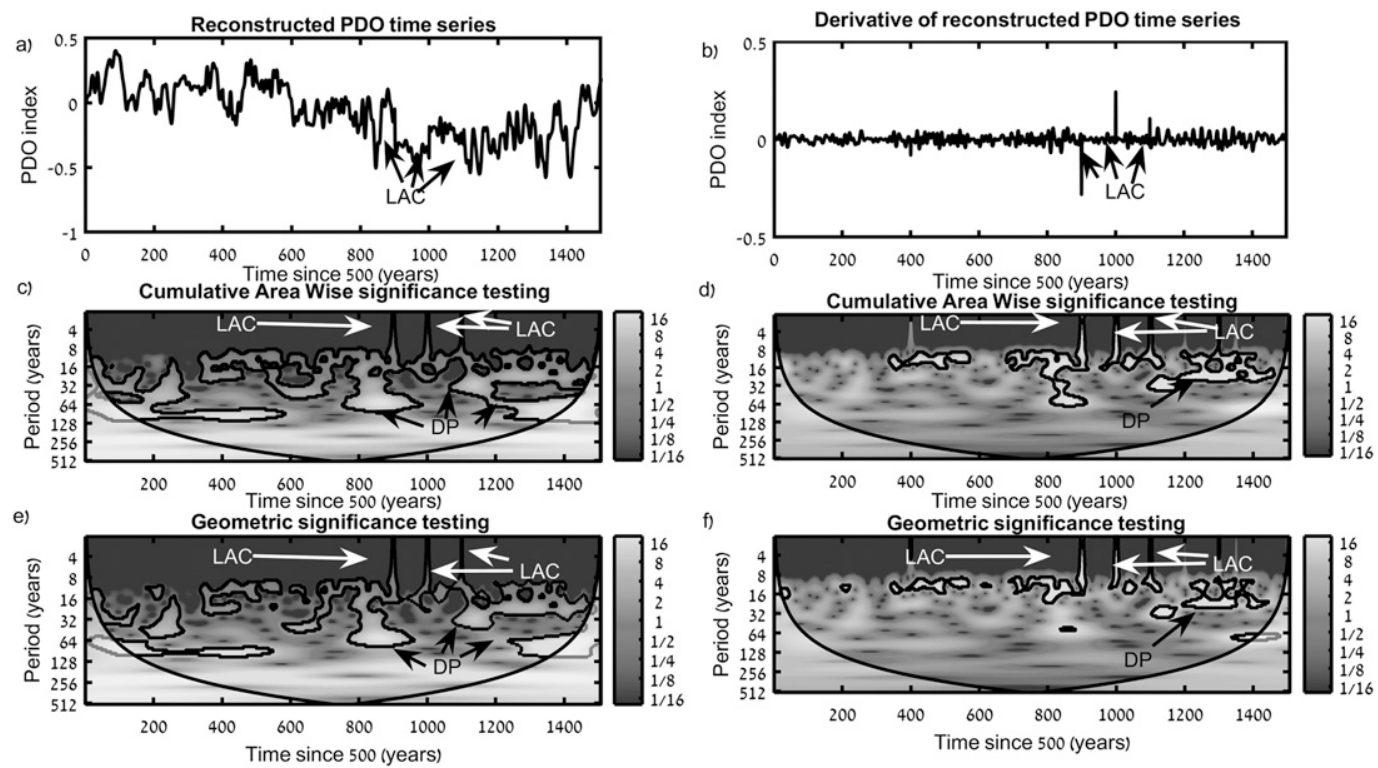

FIG. 8. As in Fig. 3, but for the reconstructed PDO (Mann et al. 2009). 

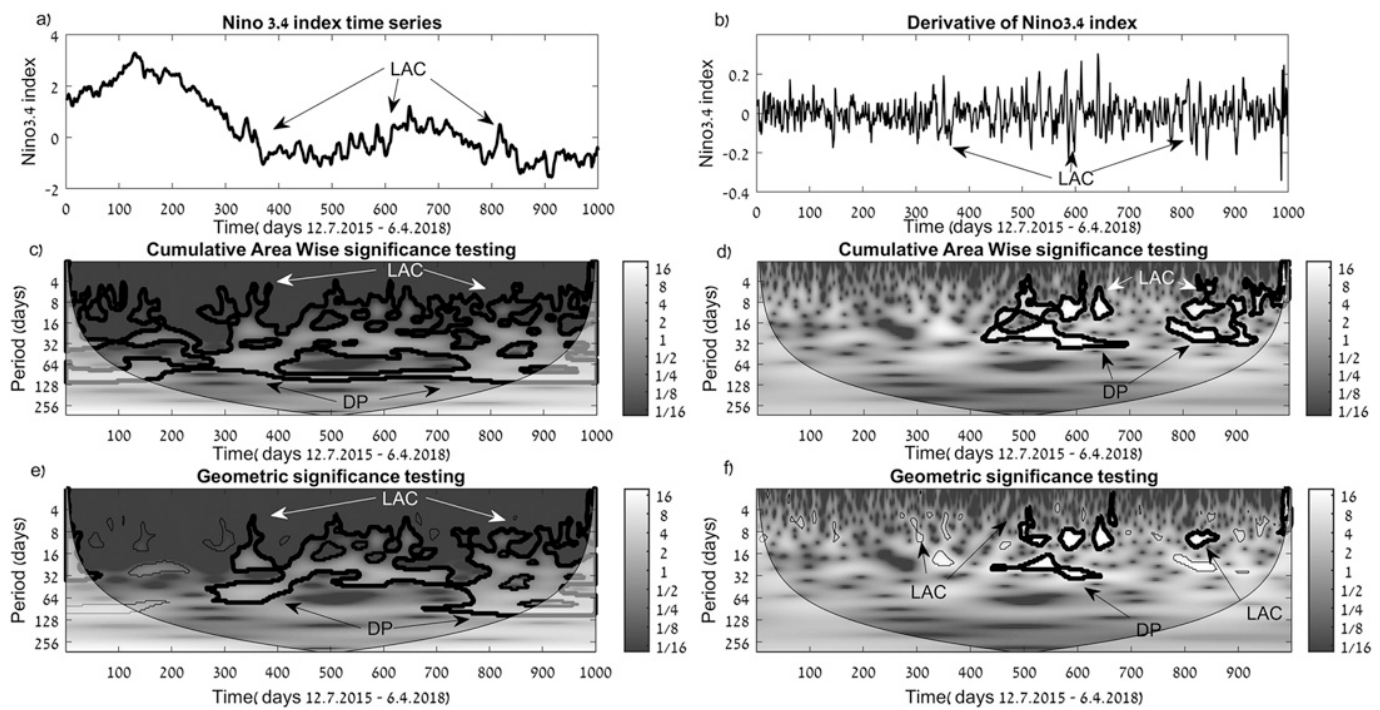

FIG. 9. As in Fig. 3, but for the Niño-3.4 index for 12 Jul 2015-6 Apr 2018.

stratospheric aerosol optical depth attributed to volcanic eruptions (Bourassa et al. 2012). Two major local abrupt changes that coincide with the large volcanic eruptions of Krakatoa in 1883 and of El Chichón and Mount Pinatubo in 1982 and 1991, respectively, produce DPs at the $10-12 \mathrm{yr}$ periods (Fig. 4). The CWT accurately detects the local abrupt change location on highfrequency scales (panels $\mathrm{c}$ and e in Figs. 3-10). However, in the lower frequencies, it is seen that the patch expands in time and results in a lower-frequency band in the wavelet power spectrum that may be erroneously interpreted as indication of a DP in the time series.
Furthermore, it seems that the cumulative areawise test is more susceptible to the emergence of lower-frequency DPs than is the geometric test.

Following the remarks at the end of section 2 with regard to the derivatives of the original time series, we analyzed also the wavelet spectrum of the derivatives. Figures $1 \mathrm{~d}, \mathrm{f}$ and $2 \mathrm{~d}$, f show that the derivative of the sine time series and the sunspot number time series, respectively, indeed leave the low-frequency periodicity intact. On the other hand, when there are local abrupt changes in the time series (e.g., Figs. 6 and 10), using the derivative emphasizes the power at higher frequencies
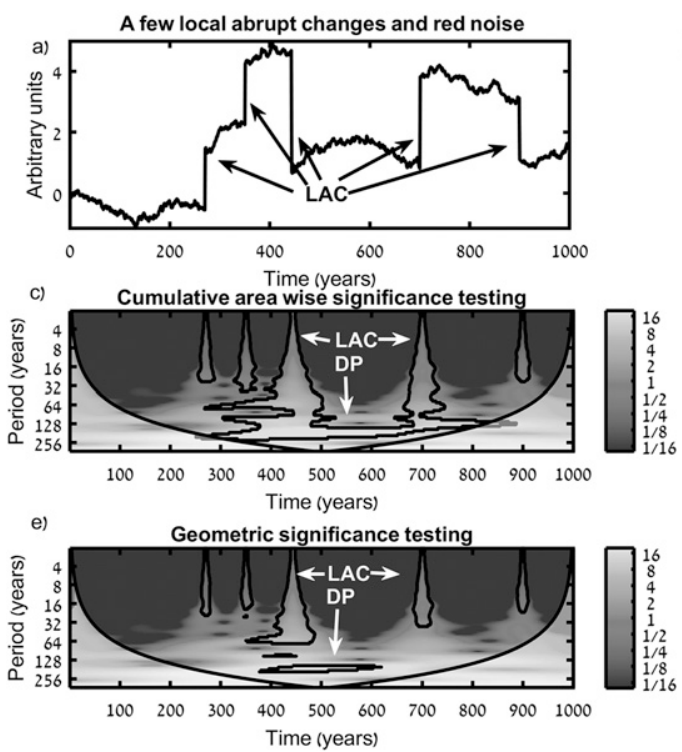
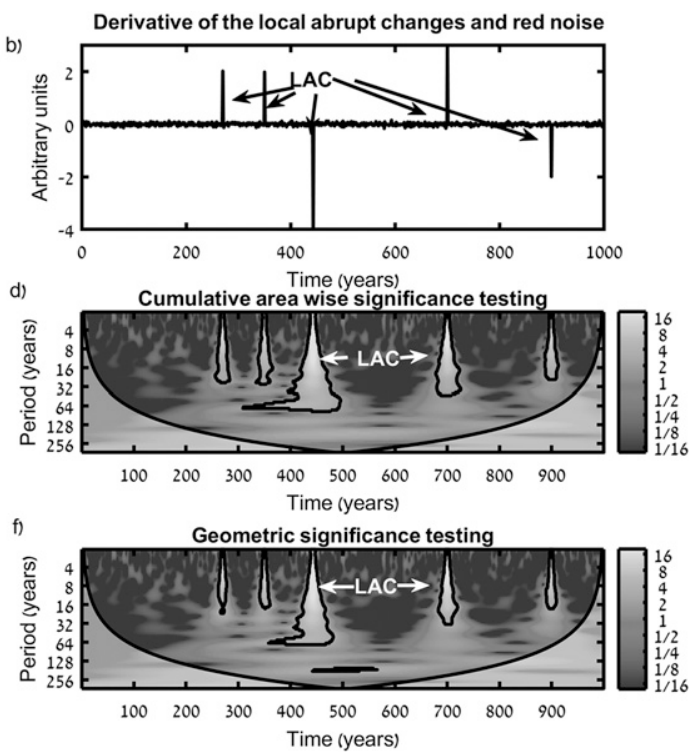

FIG. 10. As in Fig. 3, but for a time series with a few LACs added to red noise. 
in the wavelet power spectrum and reduces the power in the lower-frequency patches (Figs. 6d,f and 10d,f). However, it still does not completely prevent detection of false DPs. This is further demonstrated in real reconstructed PDO and Niño-3.4 time series (Figs. 8d,f and $9 \mathrm{~d}, \mathrm{f}$, respectively). Thus, using the derivatives is a partial remedy that does not completely neutralize the effects caused by the presence of local singularities.

The pointwise significance testing procedure (Torrence and Compo 1998) displays significant artificial lowfrequency DPs in the aforementioned time series (supplemental Fig. S2). This test does not find the highfrequency scales to be significant, in contrast to the area-based tests (Schulte et al. 2015; Schulte 2016). Therefore, the interpretation of the wavelet power spectrum using pointwise significance testing may be more misleading then the area-based tests.

Rectifying (scaling) the wavelet power spectrum may allow the comparison of spectral peaks across scales (Liu et al. 2007). However, for time series containing local abrupt changes, the cumulative areawise significance test (Schulte 2016), performed after rectifying the time series, finds the high-frequency scales to be significant but not the real DPs (supplemental Fig. S3). This is because the high- and low-frequency periods are divided by small and large numbers, respectively.

Figure S4 from the online supplemental material displays the effect of using zero padding on the synthetic time series used in this study. It shows that zero padding procedure (Fig. S4, left column) reduces edge effects in all aforementioned time series with respect to no padding (Fig. S4, right column). Therefore, it is stressed that zero padding is helpful wherever it smooths the time series at its edges, thus reducing the effect of local singularities. For example, the improvement in the boundary conditions is higher in the left side of the sine time series than in its right side (Figs. S4a,b, respectively). Accordingly, no clear recommendation can be given for climate time series since edge effects depend on the edges of the analyzed time series. It is recommended to analyze time series with different extension procedures to give a better evaluation of edge effects.

We found that the Morlet 6 mother wavelet is the most robust to the effect of local singularities on lower frequencies (Figs. 1-10), with respect to the Paul (supplemental Fig. S5) and the Dog (supplemental Fig. S6) mother wavelets. Thus, we recommend using Morlet 6 for climate time series following previous recommendations (Torrence and Compo 1998; Maraun et al. 2007; Schulte et al. 2015; Schulte 2016).

To summarize, we argue that local singularities, commonly present in climatic time series (e.g., Yosef et al. 2018) can induce low-frequency power that may be interpreted as DPs. We have demonstrated this problematic feature on synthetic time series and on real-data climatic series. However, note that any series containing local singularities would necessarily produce a DP at lower frequencies, as this is inherent to wavelet analysis (Holschneider 1995; Abramovich et al. 2000; Mallat 2008).

\section{Discussion and conclusions}

The results of using CWT for detecting periodicities in noisy climate time series might be misleading, as demonstrated here on different synthetic as well as real climatic time series. It is shown that the presence of a lower-frequency band in the wavelet power spectrum does not necessarily indicate on the presence of a true periodicity, but is often caused by local singularities in the time series (as shown in Figs. 3-6 and 8-10). Note that one can think of CWT as a series of localized bandpass filters, where low frequencies correspond to large windows in the time domain. Therefore, local singularities would be necessarily manifested in the lower frequencies domain of the wavelet power spectrum (Holschneider 1995; Abramovich et al. 2000; Mallat 2008). A finer analysis of the geometry of patch shapes is required to distinguish between a true DP and an abrupt change in a time series. The latter typically yields local maxima within a cone around its location that propagate along high frequencies in the wavelet spectrum (e.g., Mallat 2008, sections 6.1 and 6.2), whereas DPs are characterized by temporally long bands in the low-frequency domain. In this case, one could think of some length test for a patch. The problem, however, is more challenging, because there is occasionally a series of local singularities with interfering cones that might look similar to a long band in the low-frequency domain (Figs. 8-10). Here, we suggest using the derivative of a time series as an additional test to distinguish between real periodicity and low-frequency bands in the wavelet power spectrum emerging from local singularities. However, it still does not completely prevent detection of false DPs.

To summarize, in order to distinguish among various possible scenarios, a topological analysis of the entire wavelet power spectrum is required that will focus on high frequency as well. Accordingly, whenever a lowerfrequency dominant periodicity appears in the wavelet power spectrum, one should also analyze the higher frequency to distinguish between a real periodicity and an artificial one, produced by local singularities. Conelike shapes in the wavelet power spectrum, propagating from the higher to the lower frequencies, most likely indicate an artificial DP. The rigorous theory for such an analysis is a topic for further research. 
Acknowledgments. This study was partly supported by the Tel Aviv University (TAU) President and Mintz foundation and by the Porter School of Environmental Studies at TAU. This study was also partially supported by cooperation in the international virtual institute DESERVE (Dead Sea Research Venue), funded by the German Helmholtz Association, the Israel Science Foundation (ISF 1123/17), and the Mediterranean Sea Research Center of Israel (MERCI). We thank Profs. Amir Averbuch and Valeri Zheludev for most-useful discussions with regard to the basics of wavelet analysis methods (Averbuch et al. 2014, 2015).

\section{REFERENCES}

Abramovich, F., and Y. Benjamini, 1995: Thresholding of wavelet coefficients as multiple hypotheses testing procedure. Wavelets and Statistics, A. Antoniadis and G. Oppenheim, Eds., Lecture Notes in Statistics, Vol. 103, Springer-Verlag, 5-14.

_ T. C. Bailey, and T. Sapatinas, 2000: Wavelet analysis and its statistical applications. J. Roy. Stat. Soc., 49D, 1-29, https:// doi.org/10.1111/1467-9884.00216.

Addison, P. S., 2017: The Illustrated Wavelet Transform Handbook: Introductory Theory and Applications in Science, Engineering, Medicine and Finance. CRC Press, 464 pp.

Allen, M. R., and L. A. Smith, 1996: Monte Carlo SSA: Detecting irregular oscillations in the presence of colored noise. J. Climate, 9, 3373-3404, https://doi.org/10.1175/1520-0442(1996) 009<3373:MCSDIO>2.0.CO;2.

Averbuch, A. Z., P. Neittaanmäki, and V. A. Zheludev, 2014: Periodic Splines. Vol. 1, Spline and Spline Wavelet Methods with Applications to Signal and Image Processing, Springer, 496 pp.

,-- , and - 2015: Non Periodic Splines. Vol. 2, Spline and Spline Wavelet Methods with Applications to Signal and Image Processing, Springer, $426 \mathrm{pp}$.

Bourassa, A. E., and Coauthors, 2012: Large volcanic aerosol load in the stratosphere linked to Asian monsoon transport. Science, 337, 78-81, https://doi.org/10.1126/science.1219371.

Burn, M. J., and S. E. Palmer, 2015: Atlantic hurricane activity during the last millennium. Sci. Rep., 5, 12838, https://doi.org/ 10.1038/srep12838.

Cox, C. J., V. P. Walden, G. P. Compo, P. M. Rowe, M. D. Shupe, and K. Steffan, 2014: Downwelling longwave flux over summit, Greenland, 2010-2012: Analysis of surface-based observations and evaluation of ERA-Interim using wavelets. J. Geophys. Res., 119, 12317-12377, https://doi.org/10.1002/2014JD021975.

DeLong, K. L., T. M. Quinn, F. W. Taylor, K. Lin, and C. C. Shen, 2012: Sea surface temperature variability in the southwest tropical Pacific since AD 1649. Nat. Climate Change, 2, 799804, https://doi.org/10.1038/nclimate1583.

Duan, F., and Coauthors, 2014: Evidence for solar cycles in a late Holocene speleothem record from Dongge Cave, China. Sci. Rep., 4, 5159, https://doi.org/10.1038/srep05159.

Gallegati, M., 2018: A systematic wavelet-based exploratory analysis of climatic variables. Climatic Change, 148, 325-338, https://doi.org/10.1007/s10584-018-2172-8.

Gray, S. T., L. J. Graumlich, J. L. Betancourt, and G. T. Pederson, 2004: A tree-ring based reconstruction of the Atlantic Multidecadal Oscillation since 1567 A.D. Geophys. Res. Lett., 31, L12205, https://doi.org/10.1029/2004GL019932.
Holschneider, M., 1995: Wavelets-An Analysis Tool. Oxford University Press, 423 pp.

Knight, J. R., R. J. Allan, C. K. Folland, M. Vellinga, and M. E. Mann, 2005: A signature of persistent natural thermohaline circulation cycles in observed climate. Geophys. Res. Lett., 32, L20708, https://doi.org/10.1029/2005GL024233.

Kreppel, K. S., C. Caminade, S. Telfer, M. Rajerison, L. Rahalison, A. Morse, and M. Baylis, 2014: A non-stationary relationship between global climate phenomena and human plague incidence in Madagascar. PLoS Neglected Trop. Dis., 8, e3155, https://doi.org/10.1371/journal.pntd.0003155.

Lau, K. M., and H. Weng, 1995: Climate signal detection using wavelet transform: How to make a time series sing. Bull. Amer. Meteor. Soc., 76, 2391-2402, https://doi.org/10.1175/ 1520-0477(1995)076<2391:CSDUWT>2.0.CO;2.

Lee, H. F., Q. Pei, D. D. Zhang, and K. P. K. Choi, 2015: Quantifying the intra-regional precipitation variability in northwestern China over the past 1400 years. PLOS ONE, 10, e0131693, https://doi.org/10.1371/journal.pone.0131693.

Li, J., S. P. Xie, E. R. Cook, G. Huang, R. D’Arrigo, F. Liu, J. Ma, and X. T. Zheng, 2011: Inter-decadal modulation of El Niño amplitude during the last millennium. Nat. Climate Change, $\mathbf{1}$, 114-118, https://doi.org/10.1038/nclimate1086.

Lin, Y., and C. L. E. Franzke, 2015: Scale-dependency of the global mean surface temperature trend and its implication for the recent hiatus of global warming. Sci. Rep., 5, 12971, https:// doi.org/10.1038/srep12971.

Liu, Y. G., X. San Liang, and R. H. Weisberg, 2007: Rectification of the bias in the wavelet power spectrum. J. Atmos. Oceanic Technol., 24, 2093-2102, https://doi.org/10.1175/ 2007JTECHO511.1.

Magee, N. B., E. Melaas, P. M. Finocchio, M. Jardel, A. Noonan, and M. J. Lacono, 2014: Blue Hill Observatory sunshine: Assessment of climate signals in the longest continuous meteorological record in North America. Bull. Amer. Meteor. Soc., 95, 1741-1751, https://doi.org/10.1175/BAMS-D-12-00206.1.

Mallat, S., 2008: A Wavelet Tour of Signal Processing: The Sparse Way. 3rd ed. Academic Press, 832 pp.

Mann, M. E., and Coauthors, 2009: Global signatures and dynamical origins of the Little Ice Age and Medieval Climate Anomaly. Science, 326, 1256-1260, https://doi.org/10.1126/ science. 1177303.

Maraun, D., and J. Kurths, 2004: Cross wavelet analysis: Significance testing and pitfalls. Nonlinear Processes Geophys., 11, 505-514, https://doi.org/10.5194/npg-11-505-2004.

,-- , and M. Holschneider, 2007: Nonstationary Gaussian processes in wavelet domain: Synthesis, estimation, and significance testing. Phys. Rev., 75E, 016707, https://doi.org/ 10.1103/PhysRevE.75.016707.

McCabe-Glynn, S., K. R. Johnson, C. Strong, M. Berkelhammer, A. Sinha, H. Cheng, and R. L. Edwards, 2013: Variable North Pacific influence on drought in southwestern North America since AD 854. Nat. Geosci., 6, 617-621, https://doi.org/10.1038/ ngeo1862.

Novello, V. F., and Coauthors, 2016: Centennial-scale solar forcing of the South American Monsoon System recorded in stalagmites. Sci. Rep., 6, 24762, https://doi.org/10.1038/srep24762.

Pike, J., G. E. A. Swann, M. J. Leng, and M. A. Snelling, 2013: Glacial discharge along the West Antarctic peninsula during the Holocene. Nat. Geosci., 6, 199-202, https://doi.org/10.1038/ ngeo1703.

Reynolds, R. W., T. M. Smith, C. Liu, B. Dudley, K. S. Chelton, and M. G. S. Casey, 2007: Daily high-resolution-blended 
analyses for sea surface temperature. J. Climate, 20, 5473-5496, https://doi.org/10.1175/2007JCLI1824.1.

Schulte, J. A., 2016: Cumulative area wise testing in wavelet analysis and its application to geophysical time series. Nonlinear Processes Geophys., 23, 45-57, https://doi.org/10.5194/npg-23-45-2016.

, C. Duffy, and R. G. Najjar, 2015: Geometric and topologic approaches to significance testing in wavelet analysis. Nonlinear Processes Geophys., 22, 139-156, https://doi.org/10.5194/ npg-22-139-2015.

Sharma, S., J. J. Magnuson, R. D. Batt, L. A. Winslow, J. Korhonen, and Y. A. Ono, 2016: Direct observations of ice seasonality reveal changes in climate over the past 320-570 years. Sci. Rep., 6, 25061, https://doi.org/10.1038/srep25061.

SILSO World Data Center, 2019: Sunspot index and long-term solar observations, version 2.0. Royal Observatory of Belgium. Subset used: Monthly mean sunspot number, accessed 15 May 2019, http://sidc.oma.be/silso/DATA/SN_m_tot_V2.0.txt.

Soon, W., and Coauthors, 2014: A review of Holocene solar-linked climatic variation on centennial to millennial timescales:
Physical processes, interpretative frameworks and a new multiple cross-wavelet transform algorithm. Earth Sci. Rev., 134, 1-15, https://doi.org/10.1016/j.earscirev.2014.03.003.

Torrence, C., and G. P. Compo, 1998: A practical guide to wavelet analysis. Bull. Amer. Meteor. Soc., 79, 61-78, https://doi.org/ 10.1175/1520-0477(1998)079<0061:APGTWA > 2.0.CO;2.

Wright, R., M. Blackett, and C. Hill-Butler, 2015: Some observations regarding the thermal flux from Earth's erupting volcanoes for the period of 2000 to 2014. Geophys. Res. Lett., 42, 282-289, https://doi.org/10.1002/ 2014 GL061997.

Xu, D., H. Lu, G. Chu, N. Wu, C. Shen, C. Wang, and L. Mao, 2014: 500-year climate cycles stacking of recent centennial warming documented in an East Asian pollen record. Sci. Rep., 4, 3611, https://doi.org/10.1038/srep03611.

Yosef, Y., E. Aguilar, and P. Alpert, 2018: Detecting and adjusting artificial biases of long-term temperature records in Israel. Int. J. Climatol., 38, 3273-3289, https://doi.org/ $10.1002 /$ joc. 5500 . 